\title{
ARTICLE Imeglimin prevents human endothelial cell death by inhibiting mitochondrial permeability transition without inhibiting mitochondrial respiration
}

\author{
D Detaille ${ }^{1,4}$, G Vial $^{1,2,5}$, A-L Borel ${ }^{1,2,6}$, C Cottet-Rouselle $^{1,2}$, S Hallakou-Bozec $^{3}$, S Bolze ${ }^{3}$, P Fouqueray ${ }^{3}$ and E Fontaine $^{1,2}$
}

Imeglimin is the first in a new class of oral glucose-lowering agents, having recently completed its phase $2 \mathrm{~b}$ trial. As Imeglimin did show a full prevention of $\beta$-cell apoptosis, and since angiopathy represents a major complication of diabetes, we studied Imeglimin protective effects on hyperglycemia-induced death of human endothelial cells (HMEC-1). These cells were incubated in several oxidative stress environments (exposure to high glucose and oxidizing agent tert-butylhydroperoxide) which led to mitochondrial permeability transition pore (PTP) opening, cytochrome $c$ release and cell death. These events were fully prevented by Imeglimin treatment. This protective effect on cell death occurred without any effect on oxygen consumption rate, on lactate production and on cytosolic redox or phosphate potentials. Imeglimin also dramatically decreased reactive oxygen species production, inhibiting specifically reverse electron transfer through complex I. We conclude that Imeglimin prevents hyperglycemia-induced cell death in HMEC-1 through inhibition of PTP opening without inhibiting mitochondrial respiration nor affecting cellular energy status. Considering the high prevalence of macrovascular and microvascular complications in type 2 diabetic subjects, these results together suggest a potential benefit of Imeglimin in diabetic angiopathy.

Cell Death Discovery (2016) 2, 15072; doi:10.1038/cddiscovery.2015.72; published online 18 January 2016

\section{INTRODUCTION}

Imeglimin is the first in a new tetrahydrotriazine-containing class of oral glucose-lowering agents - the Glimins - and has just completed a phase 2b clinical trial (US/EU EudraCT number 2012004045-33). Several clinical trials evidenced Imeglimin efficacy on $\mathrm{HbA} 1 \mathrm{c}$ as a mono and add-on therapy, ${ }^{1-3}$ being well tolerated. Imeglimin has effects on the liver, muscle and the pancreas, ${ }^{4}$ three key organs involved in type 2 diabetes pathophysiology, through suspected mechanisms involving the mitochondria and reduced oxidative stress. Imeglimin decreases hepatic glucose production and increases muscle glucose uptake. ${ }^{4}$ Imeglimin also demonstrated increased insulin secretion in response to glucose in diabetic patients during a hyperglycemic clamp study. ${ }^{5}$ Recently, it was demonstrated in high fat high sucrose diet mice that Imeglimin normalizes glucose tolerance and insulin sensitivity by protecting mitochondrial function from oxidative stress and favoring lipid oxidation in the liver. ${ }^{6}$ In addition to its antidiabetic effects, Imeglimin also exhibited a protective effect on $\beta$-cell apoptosis induced by various stresses (high glucose or inflammatory cytokine cocktail). ${ }^{4}$

Mitochondria are involved in numerous physiological processes including energy metabolism, calcium homeostasis and programmed cell death. ${ }^{7-9}$ Several mitochondrial proteins such as cytochrome $c$ or AIF, which have no proapoptotic activity when they remain inside mitochondria, promote cell death once released into the cytosol. ${ }^{10,11}$ The permeability transition pore (PTP) is a $\mathrm{Ca}^{2+}$-sensitive mitochondrial inner membrane channel. ${ }^{12,13}$ Normally closed in order to allow ATP synthesis, permanent PTP opening leads to a drastic inhibition of ATP synthesis through the collapse of the proton-motive force, a dramatic increase in reactive oxygen species (ROS) production ${ }^{14,15}$ and a release of mitochondrial proapoptotic proteins, ${ }^{16}$ which results in cell death. ${ }^{8} \mathrm{Ca}^{2+}$ is the most important factor for PTP opening. The amount of $\mathrm{Ca}^{2+}$ required to open the pore varies according to a number of factors. PTP inhibitors and PTP inducers refer to factors that increase and decrease the amount of $\mathrm{Ca}^{2+}$ required to induce PTP opening. ${ }^{17}$ Cyclosporine A (CsA) is the reference PTP inhibitor, whereas oxidative stress is recognized to favor PTP opening. ${ }^{13}$ In several cell types, direct or indirect inhibition of respiratory chain complex I has been shown to prevent PTP opening. ${ }^{18-23}$

Angiopathy represents a major complication of diabetes that determines the quality of life and life expectancy of the diabetic patients. ${ }^{24}$ Since Imeglimin has been shown to exhibit a protective effect on glucose-induced cell death in $\beta$-cells, ${ }^{4}$ the objective of this study was to establish whether Imeglimin prevents hyperglycemia-induced cell death in human endothelial cells and to clear the mechanistic basis for its antiapoptotic property. We demonstrate that Imeglimin prevents hyperglycemia-induced cell death in HMEC-1 cells by inhibiting PTP opening without inhibiting mitochondrial respiration and strongly decreases ROS specifically produced by reverse electron transport at the mitochondrial complex I level.

\footnotetext{
${ }^{1}$ University Grenoble Alpes, LBFA, F-38000 Grenoble, France; ${ }^{2}$ INSERM, U1055, F-38000 Grenoble, France and ${ }^{3}$ Poxel, F-69000 Lyon, France.

Correspondence: E Fontaine eric.fontaine@ujf-grenoble.fr

${ }^{4}$ Current address: INSERM U1045, F-33076 Bordeaux, France.

${ }^{5}$ Current address: INSERM U1060, F-69003 Lyon, France.

${ }^{6}$ Current address: INSERM U1042, F-38000 Grenoble, France.

Received 15 November 2015; accepted 22 November 2015; Edited by A Ruffini
} 


\section{RESULTS}

Prevention of cell death by Imeglimin

In order to establish whether Imeglimin can prevent human endothelial cell death, HMEC-1 cells were exposed either to tertbutylhydroperoxide $(\mathrm{tBH})$ or high concentration of glucose, two conditions known to induce PTP opening and cell death. ${ }^{20}$ Exposure of HMEC-1 cells to $\mathrm{tBH}$ led to a significant fivefold increase in cell death that was prevented by the PTP inhibitor CsA (Figures $1 \mathrm{a}-\mathrm{c}$ ). Preincubation with Imeglimin $(10 \mathrm{mM}$ for $4 \mathrm{~h}$ or $100 \mu \mathrm{M}$ for $24 \mathrm{~h}$ ) also fully prevented $\mathrm{tBH}$-induced cell death. When HMEC-1 cells were cultured under high glucose concentration, the percentage of dead cells almost doubled after $48 \mathrm{~h}$ (Figures $1 \mathrm{~b}-\mathrm{d}$ ), and this was prevented by general antioxidant $\mathrm{N}$-acetyl cysteine and CsA, in agreement with the proposal that hyperglycemia is responsible for an oxidative stress that in turn induces PTP opening. Imeglimin similarly prevented hyperglycemiainduced endothelial cell death as shown in Figure 1.

Imeglimin prevents $\mathrm{tBH}$ - and high glucose-induced cytochrome $c$ release

To clear whether Imeglimin inhibits the cell death cascade before or after the release of mitochondrial proapoptotic proteins, we investigated the subcellular distribution of cytochrome $c$ in stressed HMEC-1. As shown in Figure 2 (left panels), cytochrome $c$ in control HMEC-1 cells was located within mitochondria. Incubation of endothelial cells in the presence of $33 \mathrm{mM}$ glucose for $48 \mathrm{~h}$, or $45 \mathrm{~min}$ exposure to $0.5 \mathrm{mM} \mathrm{tBH}$ followed by $24 \mathrm{~h}$ incubation in normal medium, induced a release of cytochrome $c$ in the cytoplasm (i.e., a presence of cytochrome $c$ outside mitochondria) in some endothelial cells (Figure 2, middle panels). Imeglimin prevented cytochrome $c$ decompartmentalization (Figure 2, right panels).
Imeglimin delays PTP opening in HMEC-1 cells

CsA inhibited PTP opening in permeabilized HMEC-1 cells as shown by an increase in calcium retention capacity (CRC) (i.e., the amount of $\mathrm{Ca}^{2+}$ required to induce PTP opening) (Figure 3b). Preincubation with $100 \mu \mathrm{M}$ Imeglimin for $24 \mathrm{~h}$ also increased the CRC, although the effect was less pronounced than the inhibition by CsA (Figure 3b). The inhibitory effect was observed when mitochondria were energized with either complex I (glutamate/malate) or complex II (succinate) substrates.

We also analyzed the effect of Imeglimin on PTP opening in intact HMEC-1 cells, which can be visualized using fluorescent compounds: calcein acetomethoxyl ester and cobalt, that do not enter mitochondria unless the PTP is open. In our experimental conditions, calcein loaded both cytosol and mitochondria but the fluorescence from cytosolic calcein was quenched by the addition of cobalt that distributes in cells but not in mitochondria. In such condition, the calcein fluorescence remains compartmentalized within mitochondria until PTP opening allows the distribution of cobalt inside mitochondria, which results in the quenching of calcein fluorescence. The addition of $\mathrm{tBH}$ led to calcein decompartmentalization (i.e., PTP opening), and this was delayed by $\operatorname{CsA} .{ }^{19,20}$ As shown in Figure 4, Imeglimin (100 $\mu \mathrm{M}$ for $24 \mathrm{~h}$ ) also delayed tBH-induced calcein decompartmentalization in intact cells. Together, these observations demonstrate that Imeglimin is a novel PTP inhibitor.

Imeglimin does not inhibit mitochondrial respiration

As shown in Table 1, Imeglimin did not inhibit rotenone-sensitive $\mathrm{NADH}$-ubiquinone oxidoreductase activity (i.e., complex I activity). Imeglimin did not affect oxygen consumption rates in intact endothelial cells (Table 2), did not stimulate glycolysis (lactate a
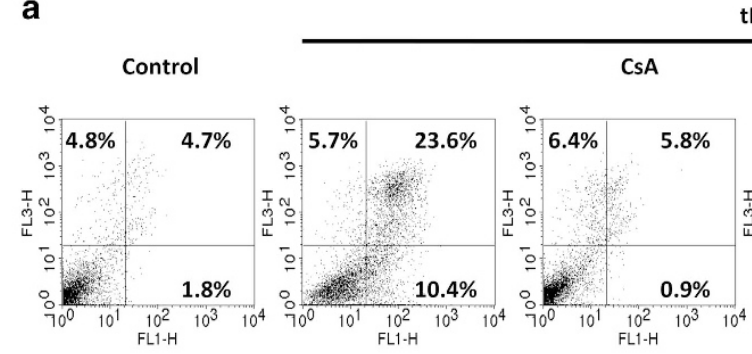

tBH

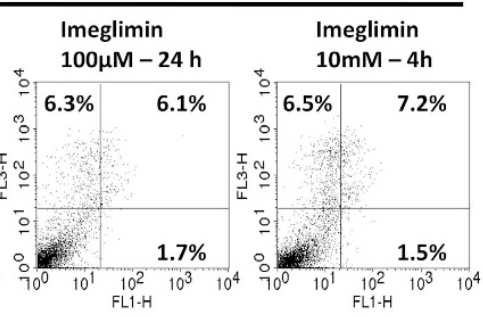

b
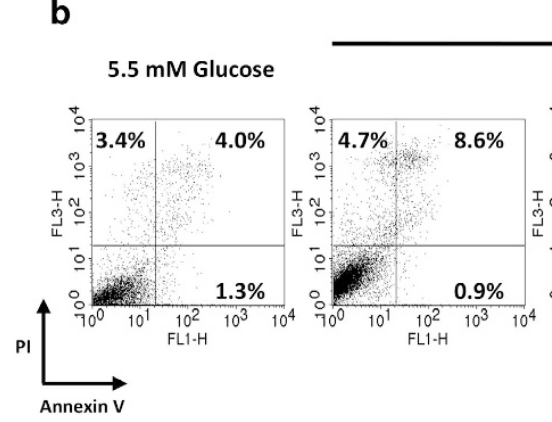

$33 \mathrm{mM}$ Glucose

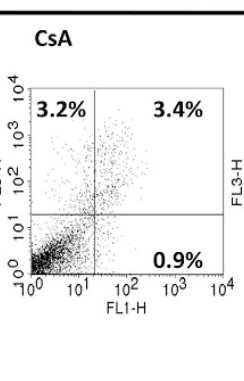

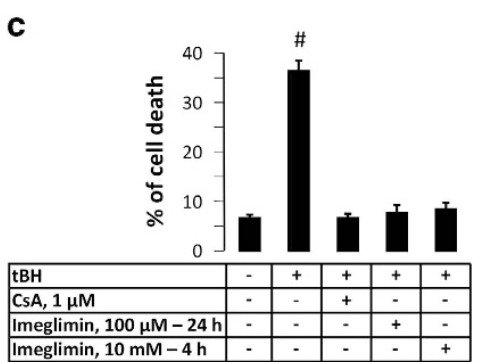

d

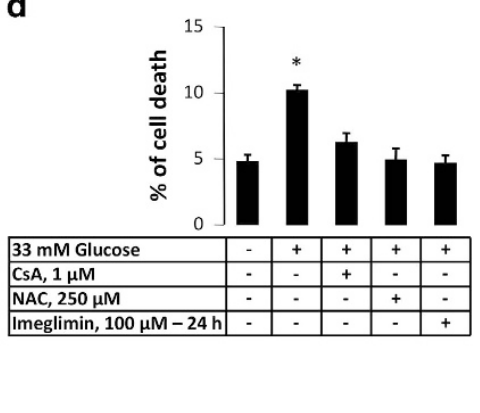

Figure 1. Effect of Imeglimin on $\mathrm{tBH}$ - or hyperglycemia-induced endothelial cell death. HMEC-1 cells incubated in the absence or presence of either $100 \mu \mathrm{M}$ Imeglimin for $24 \mathrm{~h}, 10 \mathrm{mM}$ Imeglimin for $4 \mathrm{~h}$ or $1 \mu \mathrm{M}$ CsA for $30 \mathrm{~min}$ were washed with PBS before subsequent exposure to $0.5 \mathrm{mM}$ tBH or vehicle (control) in FBS-free culture medium for $45 \mathrm{~min}$. Cells were then washed with PBS and incubated at $37^{\circ} \mathrm{C}$ for $24 \mathrm{~h}$ in a complete MCDB medium. Alternatively, HMEC-1 cells were cultured for $48 \mathrm{~h}$ in a complete MCDB medium at the indicated concentration of glucose, in the absence or presence of $1 \mu \mathrm{M} \mathrm{CsA}, 250 \mu \mathrm{M} \mathrm{N}$-acetyl cysteine (NAC) or $100 \mu \mathrm{M}$ imeglimin. Cytotoxicity was evaluated by staining cells with Alexa Fluor-conjugated annexin V and PI. (a and b) Representative data. (c and d) Percentage of dead cells (i.e., cells positive for annexin $\mathrm{V}$ or $\mathrm{PI}$ ) in five different experiments. Results are mean \pm S.E.M.; ${ }^{\#} P<0.01,{ }^{*} P<0.05$ versus control cells, paired Student's $t$-test. 

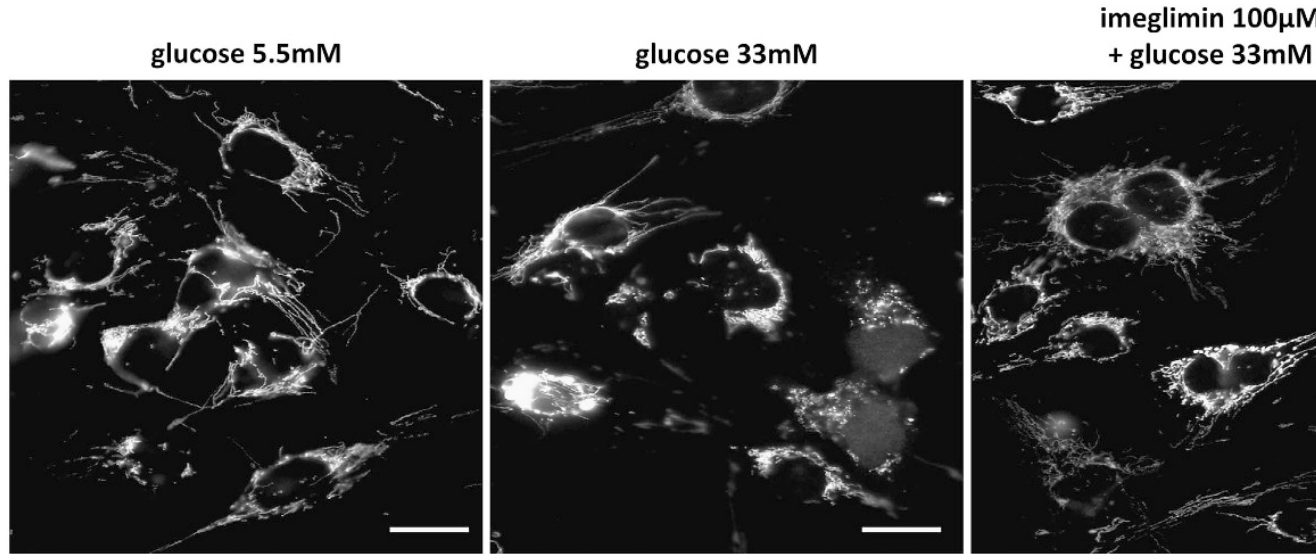

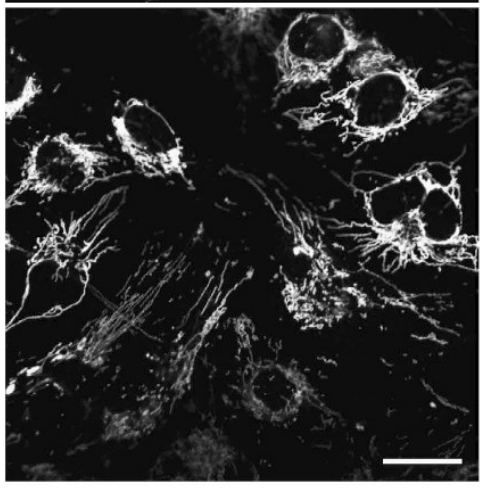

control

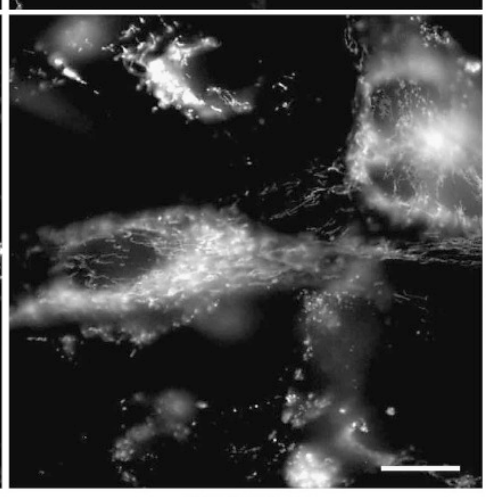

tBH $500 \mu \mathrm{M}$

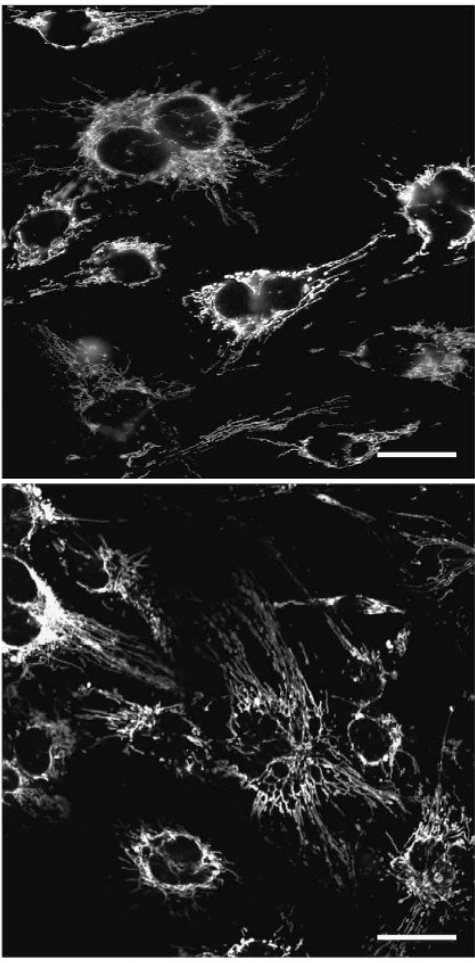

imeglimin $100 \mu \mathrm{M}$

$+\mathrm{tBH} 500 \mu \mathrm{M}$

Figure 2. Effect of Imeglimin on cytochrome $c$ distribution. HMEC-1 cells incubated in the presence or absence of Imeglimin were exposed to $\mathrm{tBH}$ or to hyperglycemic conditions as described in Figure 1 . After 24 or $48 \mathrm{~h}$ (for tBH treatment and hyperglycemia, respectively), immunostaining was performed with specific anti-cytochrome $c$ antibody. Representative data of five different experiments. Scale bar: $40 \mu$ m.

a

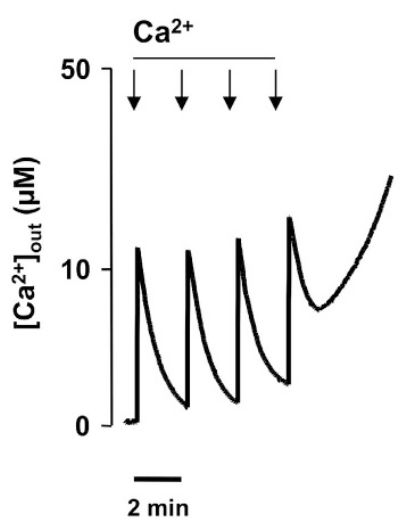

b

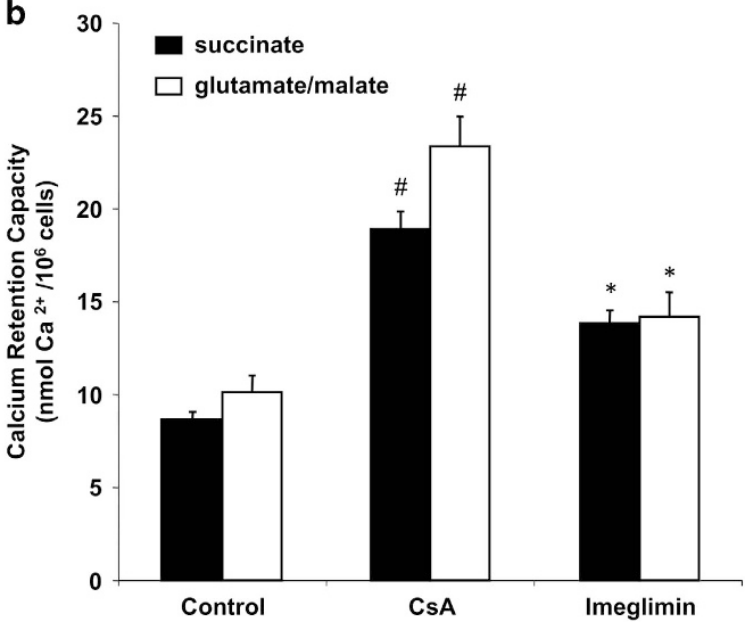

Figure 3. Effects of Imeglimin on the $\mathrm{Ca}^{2+}$ retention capacity of digitonin-permeabilized HMEC-1 cells. (a) The incubation medium contained $250 \mathrm{mM}$ sucrose, $1 \mathrm{mM}$ Pi, $10 \mathrm{mM}$ Tris-MOPS, $0.25 \mu \mathrm{M}$ Calcium Green-5N, $50 \mu \mathrm{g} / \mathrm{ml}$ digitonin and either $5 \mathrm{mM}$ succinate or $5 \mathrm{mM}$ glutamate plus $2.5 \mathrm{mM}$ malate. The final volume was $1 \mathrm{ml}\left(\mathrm{pH} \mathrm{7.35)}\right.$ at $25^{\circ} \mathrm{C}$. Experiments were started by the addition of $10^{7} \mathrm{HMEC}-1 \mathrm{cells}$. Where indicated, $12.5 \mu \mathrm{M} \mathrm{Ca}^{2+}$ pulses were added (arrows). Panel (b) represents cumulative data of five different experiments performed as described in the panel (a) after preincubation with CSA (1 $\mu \mathrm{M}$ for $30 \mathrm{~min}$ ) or Imeglimin (100 $\mu \mathrm{M}$ for $24 \mathrm{~h}$ ). Results are mean \pm S.E.M.; ${ }^{\#} P<0.01 ;{ }^{*} P<0.05$ versus control cells, paired Student's t-test.

production) and did not affect either the cytosolic redox potential (as assessed by the lactate-to-pyruvate ratio) or the phosphate potential (as assessed by the ATP/ADP ratio). In contrast, Metformin, which partly inhibits complex I (Table 1), decreased the spontaneous and stimulated (dinitrophenol (DNP)) oxygen consumption of intact cells (Table 2). Metformin increased the cytosolic redox potential and decreased the phosphate potential despite a stimulation of glycolysis (Table 2). 


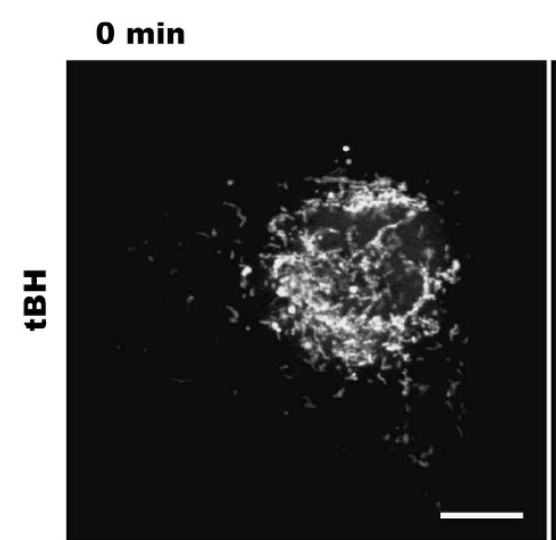

5 min
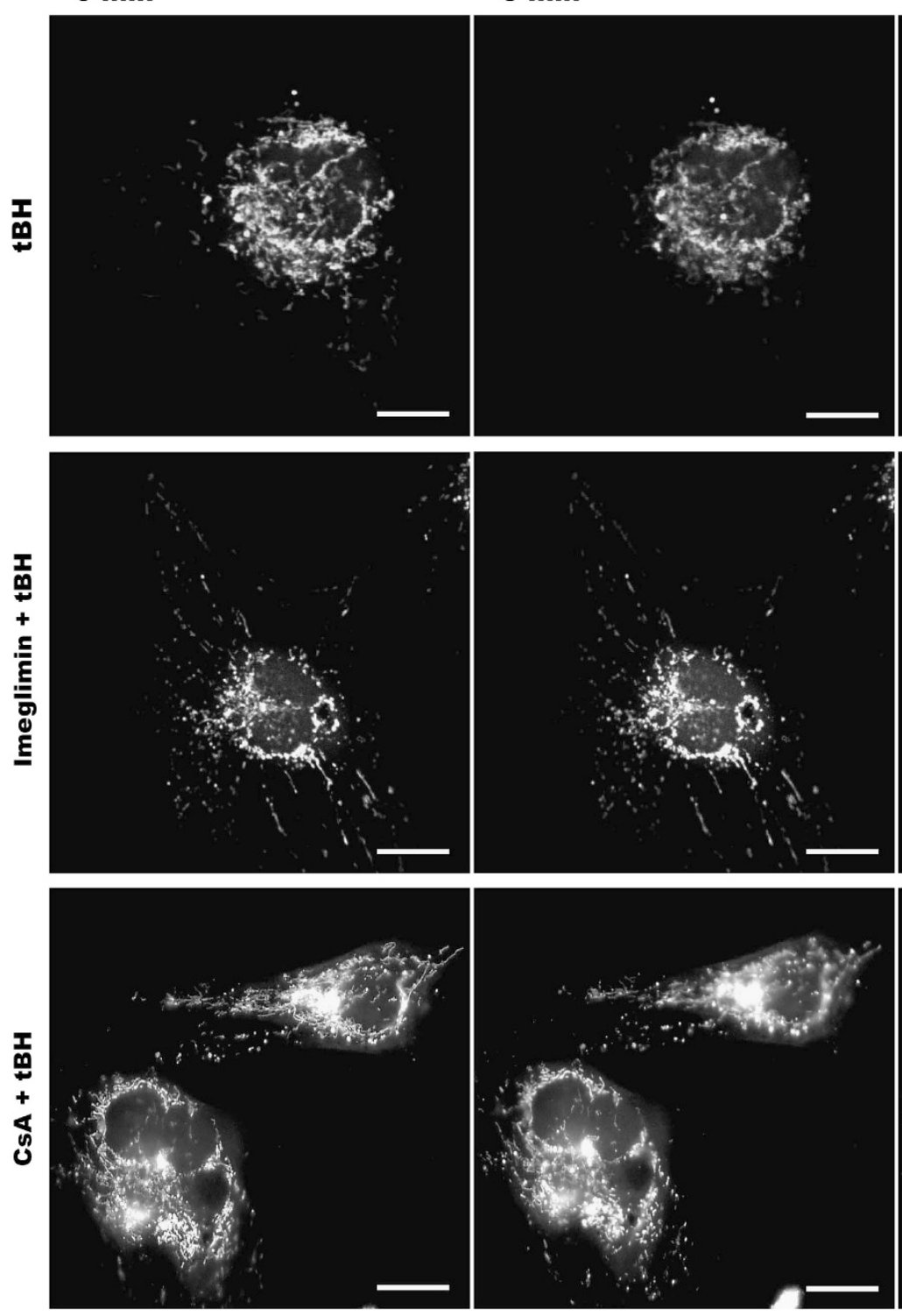

$10 \mathrm{~min}$
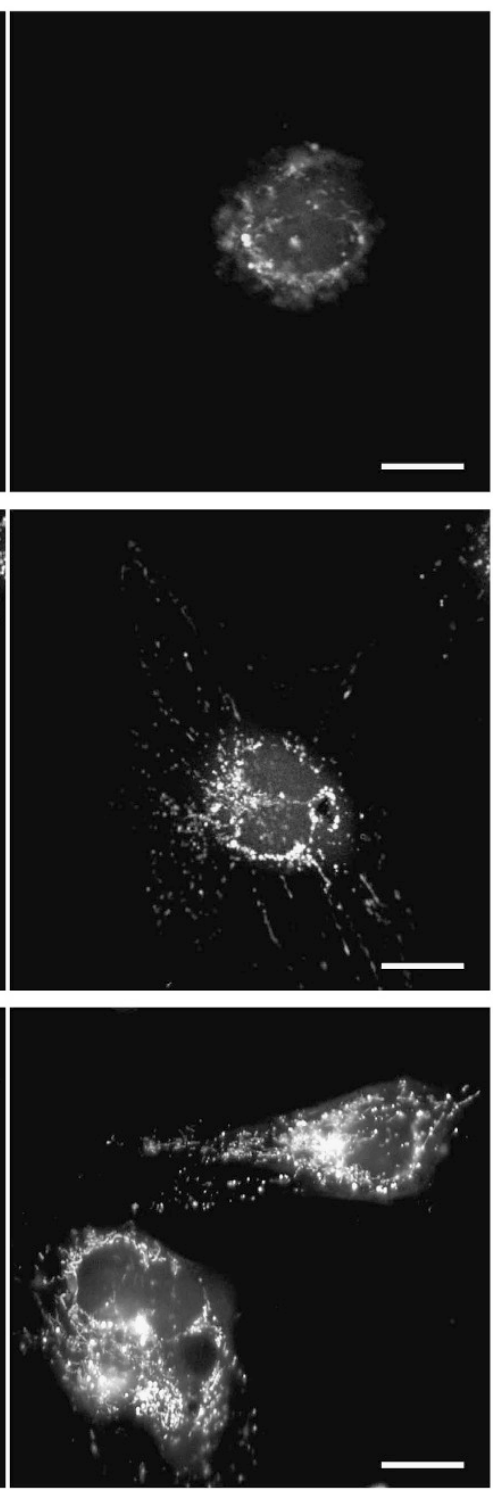

Figure 4. Effect of Imeglimin on tBH-induced PTP opening in intact HMEC-1 cells. HMEC-1 cells incubated in the absence or presence of either $100 \mu \mathrm{M}$ Imeglimin for $24 \mathrm{~h}$ or $1 \mu \mathrm{M}$ CsA for $30 \mathrm{~min}$ were co-loaded for $15 \mathrm{~min}$ with calcein plus cobalt and exposed to tBH. Images were collected every 2 min with an inverted confocal microscope, using a $\times 63$ water immersion objective. Representative data of five different experiments. Scale bar: $40 \mu \mathrm{m}$.

Table 1. Effect of Imeglimin on respiratory chain complex I activity in HMEC-1 cells

\begin{tabular}{|c|c|c|c|}
\hline Total activity $J \mathrm{NADH}\left(\mathrm{nmol} \mathrm{NADH} / \mathrm{min} / 10^{7}\right.$ cells) & $1.43 \pm 0.034$ & $1.44 \pm 0.042$ & $1.39 \pm 0.049$ \\
\hline Rotenone-sensitive Complex I activity (nmol NADH $/ \mathrm{min} / 10^{7}$ cells) & $1.035 \pm 0.027$ & $1.056 \pm 0.022$ & $0.99 \pm 0.033$ \\
\hline
\end{tabular}

HMEC-1 cells incubated in the absence or presence of either $100 \mu \mathrm{M}$ Imeglimin for $24 \mathrm{~h}$ or $10 \mathrm{mM}$ Imeglimin for $4 \mathrm{~h}$ were first permeabilized and then submitted to a hypotonic shock as described in the Material and Methods section. NADH oxidation rate was measured fluorimetrically in the presence of decylubiquinone before and after addition of rotenone. Results are means \pm S.E.M. of four independent experiments.

Imeglimin inhibits ROS production linked to reverse electron flux through complex I

As shown in Table 3, Imeglimin did not inhibit $\mathrm{H}_{2} \mathrm{O}_{2}$ production when permeabilized cells were incubated in the presence of complex I substrates (glutamate/malate) either in the resting condition or when $\mathrm{H}_{2} \mathrm{O}_{2}$ production was increased by complex I or complex III inhibitors (rotenone and antimycin A, respectively), indicating that Imeglimin does not exhibit antioxidant activity. 
Permeabilized cells were then incubated in the presence of complex II substrates alone (succinate) or in combination with glutamate and malate. In this situation (i.e., when the respiratory chain is energized with complex II substrate), most of the ROS production is due to a reverse electron flux through complex I and is abolished by complex I inhibitors. As expected, rotenone decreased $\mathrm{H}_{2} \mathrm{O}_{2}$ production when permeabilized cells were incubated in the presence of succinate. Imeglimin dramatically decreased $\mathrm{H}_{2} \mathrm{O}_{2}$ production before rotenone addition, suggesting that Imeglimin inhibited reverse electron flux through complex I.

\section{DISCUSSION}

In this study we have shown that Imeglimin exhibits antiapoptotic properties in human endothelial cells, preventing cell death induced by oxidative stresses such as hyperglycemia and $\mathrm{tBH}$. This antiapoptotic property is not restricted to human endothelial cells, since this benefit was previously reported in INS-1 cells and in rat pancreatic $\beta$-cells. ${ }^{4}$ We report for the first time that Imeglimin acts as a PTP inhibitor that prevents $\mathrm{Ca}^{2+}$-induced PTP opening in permeabilized HMEC- 1 cells and $\mathrm{tBH}$-induced PTP opening in intact HMEC-1 cells. We conclude that Imeglimin prevents cell death in HMEC-1 cells through inhibition of PTP opening.

Table 2. Comparative effects of Imeglimin and Metformin on cellular oxygen consumption $\left(\mathrm{JO}_{2}\right)$ and energy metabolism

\begin{tabular}{lccc}
\hline & Control & Imeglimin & Metformin \\
\hline $\mathrm{JO}_{2}$ (Natom O/min/mg protein) & & & \\
$\quad$ No addition & $4.9 \pm 0.14$ & $4.6 \pm 0.22$ & $3.1 \pm 0.14^{\mathrm{a}}$ \\
$\quad$ + Oligomycin & $1.6 \pm 0.04$ & $1.5 \pm 0.03$ & $1.3 \pm 0.07$ \\
$\quad+$ DNP & $9.1 \pm 0.76$ & $9.2 \pm 0.41$ & $6.1 \pm 0.51^{\mathrm{a}}$ \\
& & & \\
$\mathrm{J}$ lactate ( $\mu$ mol NADH/ & $3.65 \pm 0.27$ & $3.81 \pm 0.30$ & $4.90 \pm 0.29^{\mathrm{a}}$ \\
$30 \mathrm{~min} / \mathrm{mg}$ protein) & & & \\
Lactate/pyruvate ratio & $1.59 \pm 0.11$ & $1.65 \pm 0.13$ & $2.23 \pm 0.12^{\mathrm{a}}$ \\
ATP (nmol/mg prot) & $83.9 \pm 4.37$ & $81.6 \pm 1.7$ & $69.7 \pm 4.95^{\mathrm{a}}$ \\
ADP (nmol/mg prot) & $17.28 \pm 3.07$ & $17.38 \pm 1.38$ & $21.02 \pm 2.78$ \\
ATP/ADP & $5.09 \pm 0.70$ & $4.8 \pm 0.25$ & $3.37 \pm 0.23^{\mathrm{a}}$ \\
\hline
\end{tabular}

HMEC-1 cells incubated in the presence of either $10 \mathrm{mM}$ Imeglimin for $4 \mathrm{~h}$ or $10 \mathrm{mM}$ Metformin for $30 \mathrm{~min} . \mathrm{JO}_{2}$ was measured before and after the successive additions of oligomycin and DNP. In parallel experiment, the production of lactate and the lactate-to-pyruvate ratio, as well as the content of ATP and ADP, was determined as described in the Material and Methods section. Results are means \pm S.E.M. of four independent experiments. ${ }^{\text {a }} P<0.05$ versus control cells, paired Student's $t$-test.
Different ways to inhibit PTP opening are described in the literature. While the reference inhibitor CsA inhibits PTP opening by removing cyclophilin $D$ from the rest of the pore, ${ }^{25,26}$ we have shown over the last decade that others such as rotenone and Metformin inhibit PTP opening due to complex I inhibition. ${ }^{18,20-23,27}$ Despite the molecular nature of the PTP remains debated, these observations led us to propose a model in which PTP opening is regulated by the molecular conformation of complex I, ${ }^{23}$ this latter being known to be affected by complex I inhibitors. ${ }^{28-30}$ In the present study, Imeglimin did not inhibit either complex I activity or oxygen consumption rates in intact HMEC cells, whereas it decreased ROS production induced by reverse electron flux through complex I. Today, the mechanism by which Imeglimin inhibits PTP opening is not known but we however suggest that Imeglimin may affect the molecular conformation of complex I, without inhibiting its activity (see below).

Mitochondria are the main source of ROS production in cells. Superoxide can be generated both at respiratory chain complexes I and III and is secondarily converted in $\mathrm{H}_{2} \mathrm{O}_{2}$ by the superoxide dismutase. ${ }^{31}$ Complex $\mathrm{I}$ is a reversible proton pump that can generate superoxide during forward and reverse electron flux. ${ }^{32,33}$ The classical complex I inhibitor rotenone increases ROS production driven by a forward electron flux, whereas it decreases ROS production driven by a reverse electron flux. ${ }^{34}$ The ROS production driven by a reverse electron flux is also very sensitive to the mitochondrial membrane potential, and therefore decreases when mitochondrial respiration is increased by ATP synthesis or by uncoupling. ${ }^{35,36}$ Theoretically, the mechanism by which Imeglimin specifically decreases the ROS production driven by a reverse electron flux would imply that Imeglimin either inhibits complex I or decreases mitochondrial membrane potential (which inevitably increases mitochondrial respiration in the absence of a respiratory chain inhibitor). However, Imeglimin did not either inhibit complex I activity (Table 1) or stimulate mitochondrial respiration (Table 2). We therefore propose that Imeglimin unconventionally affects complex I functioning, inhibiting the ROS production driven by a reverse electron transfer (Table 3) without inhibiting complex I activity or forward respiratory flux.

In the present study, we have also observed that Imeglimin prevented PTP opening and subsequent HMEC-1 death in a concentration- and time-dependent manner. Indeed, the effect obtained after $4 \mathrm{~h}$ using high concentration was reached using low (clinical range) concentration after longer ( $24 \mathrm{~h}$ ) incubation time (Figures $1 \mathrm{a}-\mathrm{c}$ ), and that this response was still present when the cells were permeabilized after Imeglimin exposure, indicating the persistence of a putative mitochondrial change (Figure 3). In that respect, the case of Metformin is interesting to reconsider.

Table 3. Effect of Imeglimin on mitochondrial $\mathrm{H}_{2} \mathrm{O}_{2}$ production

$\mathrm{H}_{2} \mathrm{O}_{2}\left(\mathrm{pmol} / \mathrm{min} / 10^{7}\right.$ cells $)$

\begin{tabular}{|c|c|c|c|c|c|c|}
\hline & \multicolumn{6}{|c|}{$\mathrm{H}_{2} \mathrm{O}_{2}\left(\mathrm{pmol} / \mathrm{min} / 10^{7}\right.$ cells $)$} \\
\hline & \multicolumn{2}{|c|}{ Glutamate/malate } & \multicolumn{2}{|c|}{ Succinate } & \multicolumn{2}{|c|}{ Glutamate/malate+succinate } \\
\hline & Control & Imeglimin & Control & Imeglimin & Control & Imeglimin \\
\hline Basal & $32.3 \pm 2.4$ & $28.4 \pm 2.2$ & $25.7 \pm 3.0$ & $14.5 \pm 1.3^{\mathrm{a}}$ & $22.3 \pm 1.5$ & $15.7 \pm 1.4^{\mathrm{a}}$ \\
\hline +Rotenone & $38.3 \pm 3.2$ & $37.5 \pm 1.5$ & $10.7 \pm 1.8$ & $11.4 \pm 2.7$ & $7.8 \pm 0.9$ & $6.9 \pm 1.1$ \\
\hline \multicolumn{7}{|c|}{$\begin{array}{l}\text { HMEC- } 1 \text { cells incubated in the absence or presence of } 100 \mu \mathrm{M} \text { Imeglimin for } 24 \mathrm{~h} \text { were digitonin-permeabilized in a } \mathrm{KCl} \text { respiratory medium supplemented } \\
\text { with Amplex Red/HRP. Mitochondria were energized with glutamate/malate, succinate or both substrates (glutamate/malate plus succinate) in order to } \\
\text { measure the basal } \mathrm{H}_{2} \mathrm{O}_{2} \text { production, then } 5 \mu \mathrm{M} \text { rotenone and } 0.25 \mu \mathrm{M} \text { antimycin A were added. Results are mean } \pm \text { S.E.M. of five different experiments. } \\
\text { a } P<0.05 \text { versus control, paired Student's } t \text {-test. }\end{array}$} \\
\hline
\end{tabular}


Although it has been reported that millimolar range concentrations of Metformin directly inhibit complex I, ${ }^{37-40}$ much lower concentrations (micromolar range) are required to obtain the same result in intact cells. ${ }^{19,27,41}$ Note also that Metformin remains a mild inhibitor of complex I even at saturating concentration in shortly incubated HMEC-1 ( 30 min), yielding a similar protection upon cell viability than after long-term exposure. ${ }^{19}$ On the other hand, it has recently been shown that 2 weeks of oral gavage of rats with Metformin led to complex I inhibition in skeletal muscle ${ }^{42}$ despite Metformin blood concentration is known to remain in the micromolar range in this condition. To explain this discrepancy, it has been proposed that Metformin slowly accumulates inside mitochondria, driven by the mitochondrial membrane potential. ${ }^{38,40}$ Although theoretically plausible, Metformin pharmacokinetic studies are not consistent with a huge (millimolar range) accumulation of Metformin in organs. ${ }^{43,44}$ It was thus concluded that although a direct effect of Metformin on complex I is possible, it seems to be considerably facilitated in intact cells regardless of the exact cellular mechanisms involved in this regulation. ${ }^{41}$ As the effect of Imeglimin on HMEC-1 also requires a lot of time, this suggests that Imeglimin slowly accumulates into the cell due to the lack or deficit of transporters, or that it indirectly affects complex I through an intricate signaling mechanism which remains to be explored in more detail.

Through PTP inhibition, the antiapoptotic effects of Imeglimin on endothelial cells suggest that it may have beneficial effects for diabetes-associated angiopathy. In addition, we could also explain, at least in part, the protective role of Imeglimin on hyperglycemiainduced cell death as the consequence of lower mitochondrial ROS production. If the contribution of reverse flux-related ROS generation under normal conditions remains still questionable, it seems however to be relevant in a pathological context. Indeed, a recently published study ${ }^{45}$ showed that ischemic accumulation of succinate was responsible for mitochondrial ROS production by reverse electron transfer at respiratory complex I during reperfusion. Studies in HFHSD mice have also demonstrated that Imeglimin decreases ROS production specifically in the presence of succinate. ${ }^{6}$ In the present study, we have confirmed that Imeglimin decreases ROS production, and that it does so by decreasing reverse electron transport at mitochondrial complex I. Further investigations are however necessary to conclude about the potential benefit of Imeglimin on the cardiovascular system.

\section{CONCLUSION}

Imeglimin prevents oxidative stress- and hyperglycemia-induced cell death in HMEC-1 cells through inhibition of mitochondrial permeability transition, but without inhibiting complex I-driven mitochondrial respiration nor affecting both redox and phosphate potentials. Moreover, it has been shown that Imeglimin is able to decrease specifically ROS generation by reverse electron transport at complex I. These unique properties mean that, in addition to its antidiabetic effects, Imeglimin may help to prevent micro- and macrovascular complications in type 2 diabetes.

\section{MATERIALS AND METHODS}

\section{Cell culture conditions}

The immortalized human dermal microvascular endothelial cell line HMEC $-1^{46}$ was a kind gift from JJ Feige (CEA, Grenoble, France). The cells were grown to confluence in MCDB-131 culture medium supplemented with $15 \%$ heat-inactivated fetal bovine serum (FBS), $2 \mathrm{mM}$ L-glutamine, $50 \mathrm{Ul} / \mathrm{ml}$ penicillin, $50 \mu \mathrm{g} / \mathrm{ml}$ streptomycin, $10 \mathrm{ng} / \mathrm{ml}$ epidermal growth factor and $1 \mu \mathrm{g} / \mathrm{ml}$ hydrocortisone, and maintained in a humidified atmosphere $\left(5 \% \mathrm{CO}_{2}\right)$ at $37^{\circ} \mathrm{C}$. Cells were trypsinized, then harvested by centrifugation at 1000 r.p.m. for $10 \mathrm{~min}$.
Quantification of endothelial cell death

HMEC-1 preincubated with or without Imeglimin (10 mM for $4 \mathrm{~h}$ or $100 \mu \mathrm{M}$ for $24 \mathrm{~h}$ ) or with CSA (1 $\mu \mathrm{M}$ for $30 \mathrm{~min}$ ) were washed with PBS before subsequent exposure to $0.5 \mathrm{mM} \mathrm{tBH}$ in FBS-free culture medium for $45 \mathrm{~min}$. Cells were then washed with PBS and incubated at $37{ }^{\circ} \mathrm{C}$ for $24 \mathrm{~h}$ in a complete MCDB medium. Alternatively, cells were exposed to $5.5 \mathrm{mM}$ glucose (control cells) or to $33 \mathrm{mM}$ glucose for $48 \mathrm{~h}$. Cytotoxicity was evaluated with a double-stain system using the Annexin V-Fluoprobes 488 kit combined with propidium iodide. Data acquisition was carried out using a FACScan flow cytometer equipped with a $15 \mathrm{~mW}$ argon ion laser tuned at $488 \mathrm{~nm}$.

\section{Immunohistochemistry of cytochrome $c$}

For the visualization of cytochrome $c$ by immunohistochemistry, cells were fixed in 3.7\% paraformaldehyde/PBS for $20 \mathrm{~min}$, permeabilized in $0.2 \%$ Triton $\mathrm{X}-100$ for $5 \mathrm{~min}$ and blocked in 2\% bovine serum albumin/PBS for $1 \mathrm{~h}$. Cells were then incubated with a monoclonal anti-cytochrome $c$ (clone $6 \mathrm{H} 2 . \mathrm{B} 4$ ) for $2 \mathrm{~h}$ and exposed to Oregon green-labeled fluorescent secondary antibody in the dark at room temperature for $1 \mathrm{~h}$. After final washes, $0.2 \mathrm{M}$ Tris- $\mathrm{HCl} \mathrm{pH}$ 7.8, 90\% glycerol and 2.3\% 1,4-diazobicyclo-[2.2.2]-octane (DABCO, antifading agent) was applied to cell preparations. Imaging was performed by confocal microscopy using a $\times 63 / 1.20$ Plan Apo water immersion objective. Laser excitation was $488 \mathrm{~nm}$, with a fluorescence emission adjusted with acousto optical beam splitter (AOBS) at 515-535 nm. Eight fields, randomly chosen with phase-contrast microscopy and containing about 10-20 cells each, were scanned by glass coverslips.

\section{Determination of mitochondrial permeability transition in} permeabilized cells

PTP opening was evaluated in vitro by measuring the CRC of digitoninpermeabilized HMEC-1 cells. Cells were permeabilized immediately before use by incubation under stirring at $25^{\circ} \mathrm{C}$ in a medium containing $250 \mathrm{mM}$ sucrose, $10 \mathrm{mM}$ Tris-MOPS (3-( $N$-morpholino)propanesulfonic acid), $1 \mathrm{mM} \mathrm{Pi}$ (inorganic phosphate)-Tris, $50 \mu \mathrm{g} / \mathrm{ml}$ digitonin, and either $5 \mathrm{mM}$ succinate or $5 \mathrm{mM} / 2.5 \mathrm{mM}$ glutamate/malate $(\mathrm{pH} 7.35)$. The measurement of extra-mitochondrial calcium concentration was carried out fluorimetrically at $25^{\circ} \mathrm{C}$ with a PTI Quantamaster spectrofluorometer equipped with magnetic stirring and thermostatic control in the presence of $0.25 \mu \mathrm{M}$ calcium green $5 \mathrm{~N}$ (excitation and emission wavelengths were set at 506 and $532 \mathrm{~nm}$, respectively). Calcium loading was performed by repetitive additions of $12.5 \mu \mathrm{M}$ calcium until PTP opening occurred.

\section{Determination of mitochondrial permeability transition in intact} cells

PTP opening was also observed in vivo using calcein/cobalt staining in living cells. ${ }^{47}$ HMEC-1 cells grown for $48 \mathrm{~h}$ on $22 \mathrm{~mm}$ diameter glass coverslips were exposed for $15 \mathrm{~min}$ at $37{ }^{\circ} \mathrm{C}$ to PBS medium supplemented with $5 \mathrm{mM}$ glucose, $0.35 \mathrm{mM}$ pyruvate, $1 \mathrm{mM} \mathrm{CoCl}$ and $1 \mu \mathrm{M}$ calceinaceto-methoxyl ester. After loading, cells were washed free of calcein and $\mathrm{CoCl}_{2}$ and further incubated for $20 \mathrm{~min}$ at $37^{\circ} \mathrm{C}$ in PBS/glucose/pyruvate medium. Cell imaging was performed with a LEICA TCS SP2 inverted laser confocal microscope, using a $\times 63 / 1.20$ Plan Apo water immersion objective. Laser excitation was $488 \mathrm{~nm}$, with a fluorescence emission adjusted with AOBS at $506-541 \mathrm{~nm}$. Cell images were collected every minute with a constant exposure time.

Assay of isolated respiratory chain complex I

Confluent monolayers of HMEC-1 cells were incubated in the absence or presence of Imeglimin (10 mM for $4 \mathrm{~h}$ or $100 \mu \mathrm{M}$ for $24 \mathrm{~h}$ ). Cells were harvested, permeabilized in a digitonin-containing cold buffer and then spun down (10 000 r.p.m. for $10 \mathrm{~min}$ ) to eliminate cytosolic contaminating enzyme activities. Permeabilized cells were placed in $800 \mu \mathrm{l} \mathrm{H}_{2} \mathrm{O}$ in a stirred glass cuvette for $2 \mathrm{~min}$ at $30^{\circ} \mathrm{C}$ to break mitochondrial membranes by hypotonic shock. Next, $200 \mu \mathrm{l}$ Tris solution $(50 \mathrm{mM}, \mathrm{pH}$ 8.0) containing $50 \mu \mathrm{M} \mathrm{NADH}$ was added for $1 \mathrm{~min}$, and the reaction started by adding $50 \mu \mathrm{M}$ decylubiquinone as a final electron acceptor. NADH oxidation rate was measured fluorimetrically (excitation-emission, $340-460 \mathrm{~nm}$ ). The rotenone-sensitive complex I activity was obtained after subtraction of the remaining signal in the presence of $10 \mu \mathrm{M}$ rotenone. 
Measurement of oxygen consumption rate in intact endothelial cells

After preincubation in MCDB-131 medium with or without Imeglimin or Metformin, intact HMEC-1 cells $\left(1.5 \times 10^{7}\right.$ cells $\left./ \mathrm{ml}\right)$ were placed at $37^{\circ} \mathrm{C}$ in an oxygraph vessel equipped with a Clark oxygen electrode and filled up with MCDB-131 culture medium devoid of any supplements. The rate of oxygen consumption $\left(\mathrm{JO}_{2}\right)$ was measured, then $2 \mu \mathrm{g} / \mathrm{ml}$ oligomycin (in order to inhibit ATP synthesis) and $125 \mu \mathrm{M}$ DNP (in order to uncouple mitochondria) were successively added to the incubation medium.

\section{Assessment of energy metabolism}

Cells were lysed by adding perchloric acid, which destroyed the enzymes but not the metabolites. After centrifugation at 12000 r.p.m. for 5 min and neutralization of the supernatant with $\mathrm{KOH}(2 \mathrm{M}) / \mathrm{MOPS}(0.3 \mathrm{M})$, lactate and pyruvate were enzymatically measured while adenine nucleotides were measured by HPLC as previously described. ${ }^{48}$ The lactate/pyruvate ratio, which is proportional to the cytosolic NADH/NAD ratio, was taken as an index of the cytosolic redox potential.

\section{Detection of $\mathrm{H}_{2} \mathrm{O}_{2}$ production}

The rate of $\mathrm{H}_{2} \mathrm{O}_{2}$ formation in permeabilized cells was measured fluorimetrically using amplex red (excitation-emission, $560-583 \mathrm{~nm}$ ) in the presence of horseradish peroxidase. $2.5 \times 10^{7}$ permeabilized cells were incubated at $30^{\circ} \mathrm{C}$ in a medium containing $125 \mathrm{mM} \mathrm{KCl}, 20 \mathrm{mM}$ Tris- $\mathrm{HCl}, 1 \mathrm{mM} \mathrm{EGTA}, 2.5 \mathrm{mM}$ PiTris $(\mathrm{pH} 7.35)$ and $10 \mu \mathrm{M}$ oligomycin. $\mathrm{H}_{2} \mathrm{O}_{2}$ production was initiated with glutamate/malate or succinate as respiratory substrates, then $5 \mu \mathrm{M}$ rotenone and $0.25 \mu \mathrm{M}$ antimycin $\mathrm{A}$ were sequentially added to measure the maximum rate of $\mathrm{H}_{2} \mathrm{O}_{2}$ production of complexes I and I+III, respectively.

\section{Statistical analysis}

Data are presented as means+S.E.M. Statistical significance of differences was analyzed using the paired Student's $t$-test.

\section{ABBREVIATIONS}

PTP, permeability transition pore; AIF, apoptosis inducing factor; HMEC-1, human endothelial cells; CsA, Cyclosporine $A ;$ ROS, reactive oxygen species; $\mathrm{tBH}$, tert-butyl hydroperoxide; NAC, N-acetyl cysteine; FBS, fetal bovine serum; BSA, bovine serum albumin; AOBS, acousto optical beam splitter; CRC, calcium retention capacity; MOPS, 3-[N-morpholino]propanesulfonic acid; $\mathrm{JO}_{2}$, rate of oxygen consumption; DNP, dinitrophenol; PCA, perchloric acid.

\section{COMPETING INTERESTS}

This work has been funded by Poxel SA. The funding body played role in the design of the study, in the writing of the manuscript and in the decision to submit the manuscript for publication. Dr. Detaille, Dr. Vial, Dr. Borel, Ms. Cottet-Rouselle and Prof. Fontaine declare no other potential conflict of interest. Dr. Hallakou-Bozec, Dr. Bolze and Dr. Fouqueray are employee by Poxel SA.

\section{REFERENCES}

1 Fouqueray P, Pirags V, Diamant M, Schernthaner G, Lebovitz HE, Inzucchi SE et al. The efficacy and safety of imeglimin as add-on therapy in patients with type 2 diabetes inadequately controlled with sitagliptin monotherapy. Diabetes Care 2014; 37: 1924-1930.

2 Fouqueray P, Pirags V, Inzucchi SE, Bailey CJ, Schernthaner G, Diamant M et al. The efficacy and safety of imeglimin as add-on therapy in patients with type 2 diabetes inadequately controlled with metformin monotherapy. Diabetes Care 2013; 36: $565-568$.

3 Pirags V, Lebovitz $\mathrm{H}$, Fouqueray P. Imeglimin, a novel glimin oral antidiabetic, exhibits a good efficacy and safety profile in type 2 diabetic patients. Diabetes Obes Metab 2012; 14: 852-858.

4 Fouqueray $\mathrm{P}$, Leverve $\mathrm{X}$, Fontaine $\mathrm{E}$, Baquié $\mathrm{M}$, Wollheim $\mathrm{C}$, Lebovitz $\mathrm{HE}$ et al. Imeglimin - a new oral anti-diabetic that targets the three key defects of type 2 diabetes. J Diabetes Metab 2011; 2: 126.

5 Pacini G, Mari A, Fouqueray P, Bolze S, Roden M. Imeglimin increases glucosedependent insulin secretion and improves beta-cell function in patients with type 2 diabetes. Diabetes Obes Metab 2015; 17: 541-545.

6 Vial G, Chauvin MA, Bendridi N, Durand A, Meugnier E, Madec AM et al. Imeglimin normalizes glucose tolerance and insulin sensitivity and improves mitochondrial function in liver of a high-fat, high-sucrose diet mice model. Diabetes 2015; 64: 2254-2264.

7 Bouchier-Hayes L, Lartigue L, Newmeyer DD. Mitochondria: pharmacological manipulation of cell death. J Clin Invest 2005; 115: 2640-2647.

8 Desagher S, Martinou JC. Mitochondria as the central control point of apoptosis. Trends Cell Biol 2000; 10: 369-377.

9 Duchen MR. Mitochondria and calcium: from cell signalling to cell death. J Physiol 2000; 529(Pt 1): 57-68.

10 Green DR, Kroemer G. The pathophysiology of mitochondrial cell death. Science 2004; 305: 626-629.

11 Saelens X, Festjens N, Vande Walle L, van Gurp M, van Loo G, Vandenabeele P. Toxic proteins released from mitochondria in cell death. Oncogene 2004; 23: 2861-2874.

12 Bernardi P, Krauskopf A, Basso E, Petronilli V, Blachly-Dyson E, Di Lisa F et al. The mitochondrial permeability transition from in vitro artifact to disease target. FEBS J 2006; 273: 2077-2099.

13 Zoratti M, Szabo I. The mitochondrial permeability transition. Biochim Biophys Acta 1995; 1241: 139-176.

14 Batandier C, Leverve X, Fontaine E. Opening of the mitochondrial permeability transition pore induces reactive oxygen species production at the level of the respiratory chain complex I. J Biol Chem 2004; 279: 17197-17204.

15 Zorov DB, Filburn CR, Klotz LO, Zweier JL, Sollott SJ. Reactive oxygen species (ROS)-induced ROS release: a new phenomenon accompanying induction of the mitochondrial permeability transition in cardiac myocytes. J Exp Med 2000; 192: 1001-1014.

16 Kantrow SP, Piantadosi CA. Release of cytochrome c from liver mitochondria during permeability transition. Biochem Biophys Res Commun 1997; 232: 669-671.

17 Bernardi P. Mitochondrial transport of cations: channels, exchangers, and permeability transition. Physiol Rev 1999; 79: 1127-1155.

18 Chauvin C, De Oliveira F, Ronot X, Mousseau M, Leverve X, Fontaine E. Rotenone inhibits the mitochondrial permeability transition-induced cell death in U937 and KB cells. J Biol Chem 2001; 276: 41394-41398.

19 Detaille D, Guigas B, Chauvin C, Batandier C, Fontaine E, Wiernsperger N et al. Metformin prevents high-glucose-induced endothelial cell death through a mitochondrial permeability transition-dependent process. Diabetes 2005; 54: 2179-2187.

20 Guigas B, Detaille D, Chauvin C, Batandier C, De Oliveira F, Fontaine E et al. Metformin inhibits mitochondrial permeability transition and cell death: a pharmacological in vitro study. Biochem J 2004; 382(Pt 3): 877-884.

21 Lablanche S, Cottet-Rousselle C, Argaud L, Laporte C, Lamarche F, Richard MJ et al. Respective effects of oxygen and energy substrate deprivation on beta cell viability. Biochim Biophys Acta 2015; 1847: 629-639.

22 Lablanche S, Cottet-Rousselle C, Lamarche F, Benhamou PY, Halimi S, Leverve X et al. Protection of pancreatic INS-1 beta-cells from glucose- and fructose-induced cell death by inhibiting mitochondrial permeability transition with cyclosporin $A$ or metformin. Cell Death Dis 2011; 2: e134.

23 Li B, Chauvin C, De Paulis D, De Oliveira F, Gharib A, Vial G et al. Inhibition of complex I regulates the mitochondrial permeability transition through a phosphate-sensitive inhibitory site masked by cyclophilin D. Biochim Biophys Acta 2012; 1817: 1628-1634.

24 Mazzone T, Chait A, Plutzky J. Cardiovascular disease risk in type 2 diabetes mellitus: insights from mechanistic studies. Lancet 2008; 371: 1800-1809.

25 Connern $\mathrm{CP}$, Halestrap AP. Chaotropic agents and increased matrix volume enhance binding of mitochondrial cyclophilin to the inner mitochondrial membrane and sensitize the mitochondrial permeability transition to [Ca2+]. Biochemistry 1996; 35: 8172-8180.

26 Nicolli A, Basso E, Petronilli V, Wenger RM, Bernardi P. Interactions of cyclophilin with the mitochondrial inner membrane and regulation of the permeability transition pore, and cyclosporin A-sensitive channel. J Biol Chem 1996; 271: 2185-2192.

27 Detaille D, Guigas B, Leverve X, Wiernsperger N, Devos P. Obligatory role of membrane events in the regulatory effect of metformin on the respiratory chain function. Biochem Pharmacol 2002; 63: 1259-1272.

28 Grivennikova VG, Kapustin AN, Vinogradov AD. Catalytic activity of $\mathrm{NADH}$-ubiquinone oxidoreductase (complex I) in intact mitochondria. evidence for the slow active/inactive transition. I Biol Chem 2001; 276: 9038-9044.

29 Grivennikova VG, Kotlyar AB, Karliner JS, Cecchini G, Vinogradov AD. Redoxdependent change of nucleotide affinity to the active site of the mammalian complex I. Biochemistry 2007; 46: 10971-10978.

30 Vinogradov AD, Grivennikova VG. The mitochondrial complex I: progress in understanding of catalytic properties. IUBMB Life 2001; 52: 129-134.

31 Cadenas E, Boveris A, Ragan $\mathrm{Cl}$, Stoppani AO. Production of superoxide radicals and hydrogen peroxide by $\mathrm{NADH}$-ubiquinone reductase and ubiquinol- 
cytochrome c reductase from beef-heart mitochondria. Arch Biochem Biophys 1977; 180: 248-257.

32 Boveris A, Chance B. The mitochondrial generation of hydrogen peroxide. General properties and effect of hyperbaric oxygen. Biochem J 1973; 134: 707-716.

33 Korshunov SS, Skulachev VP, Starkov AA. High protonic potential actuates a mechanism of production of reactive oxygen species in mitochondria. FEBS Lett 1997; 416: 15-18.

34 Turrens JF. Superoxide production by the mitochondrial respiratory chain. Biosci Rep 1997; 17: 3-8.

35 Batandier C, Guigas B, Detaille D, El-Mir MY, Fontaine E, Rigoulet M et al. The ROS production induced by a reverse-electron flux at respiratorychain complex 1 is hampered by metformin. J Bioenerg Biomembr 2006; 38: 33-42.

36 Votyakova TV, Reynolds IJ. DeltaPsi(m)-Dependent and -independent production of reactive oxygen species by rat brain mitochondria. J Neurochem 2001; 79: 266-277.

37 Wheaton WW, Weinberg SE, Hamanaka RB, Soberanes S, Sullivan LB, Anso E et al. Metformin inhibits mitochondrial complex I of cancer cells to reduce tumorigenesis. elife 2014; 3: e02242.

38 Bridges HR, Jones AJ, Pollak MN, Hirst J. Effects of metformin and other biguanides on oxidative phosphorylation in mitochondria. Biochem $J$ 2014; 462: 475-487.

39 Palenickova E, Cahova M, Drahota Z, Kazdova L, Kalous M. Inhibitory effect of metformin on oxidation of $\mathrm{NADH}$-dependent substrates in rat liver homogenate. Physiol res 2011; 60: 835-839.

40 Owen MR, Doran E, Halestrap AP. Evidence that metformin exerts its anti-diabetic effects through inhibition of complex 1 of the mitochondrial respiratory chain. Biochem J 2000; 348(Pt 3): 607-614.

41 El-Mir MY, Nogueira V, Fontaine E, Averet N, Rigoulet M, Leverve X. Dimethylbiguanide inhibits cell respiration via an indirect effect targeted on the respiratory chain complex I. J Biol Chem 2000; 275: 223-228.
42 Wessels B, Ciapaite J, van den Broek NM, Nicolay K, Prompers JJ. Metformin impairs mitochondrial function in skeletal muscle of both lean and diabetic rats in a dose-dependent manner. PloS One 2014; 9: e100525.

43 Higgins JW, Bedwell DW, Zamek-Gliszczynski MJ. Ablation of both organic cation transporter (OCT)1 and OCT2 alters metformin pharmacokinetics but has no effect on tissue drug exposure and pharmacodynamics. Drug Metab Dispos 2012; 40: $1170-1177$.

44 Wang DS, Jonker JW, Kato $Y$, Kusuhara H, Schinkel AH, Sugiyama Y. Involvement of organic cation transporter 1 in hepatic and intestinal distribution of metformin. J Pharmacol Exp Ther 2002; 302: 510-515.

45 Chouchani ET, Pell VR, Gaude E, Aksentijevic D, Sundier SY, Robb EL et al. Ischaemic accumulation of succinate controls reperfusion injury through mitochondrial ROS. Nature 2014; 515: 431-435.

46 Ades EW, Candal FJ, Swerlick RA, George VG, Summers S, Bosse DC et al. HMEC-1: establishment of an immortalized human microvascular endothelial cell line. $J$ Invest Dermatol 1992; 99: 683-690.

47 Petronilli V, Miotto G, Canton M, Brini M, Colonna R, Bernardi P et al. Transient and long-lasting openings of the mitochondrial permeability transition pore can be monitored directly in intact cells by changes in mitochondrial calcein fluorescence. Biophys J 1999; 76: 725-734.

48 Argaud D, Roth H, Wiernsperger N, Leverve XM. Metformin decreases gluconeogenesis by enhancing the pyruvate kinase flux in isolated rat hepatocytes. Eur J Biochem/FEBS 1993; 213: 1341-1348.

(i) This work is licensed under a Creative Commons Attribution 4.0 International License. The images or other third party material in this article are included in the article's Creative Commons license, unless indicated otherwise in the credit line; if the material is not included under the Creative Commons license, users will need to obtain permission from the license holder to reproduce the material. To view a copy of this license, visit http://creativecommons.org/licenses/ by/4.0/ 